\title{
New Radial Velocities and the Barnes-Evans Method
}

\author{
Robert Hindsley ${ }^{1}$ and R.A. Bell ${ }^{2}$, \\ ${ }^{1}$ U.S. Naval Observatory, Washington D.C. \\ ${ }^{2}$ University of Maryland
}

\begin{abstract}
New radial velocities by Butler are used to recalculate the absolute magnitudes of Eta Aquilae and X Cygni using the Barnes-Evans method. The new velocities have little effect on the result for Eta Aquilae, but the absolute magnitude of $\mathrm{X}$ Cygni is found to be $0.40 \mathrm{mag}$ fainter than that obtained earlier.
\end{abstract}

Butler (these Proceedings) has measured the radial velocities of four Cepheids (FF Aql, Delta Cep, Eta Aql, and X Cyg) using an iodine cell to give reference wavelengths for the spectral lines. With this technique the standard error of a single measurement is a few hundred metres per second. The ratio of the uncertainty in each velocity point to the full amplitude of the radial velocity variation now matches the corresponding ratio for the $V$ magnitude light curve.

For Eta Aquilae, the Fourier decomposition of the radial velocities of Jacobsen and Wallerstein (1981, Publ. A.S.P, 93, 481) and Evans (1976, Ap. J. Suppl., 32, 399) were compared to the decomposition obtained by combining these data with the data of Butler. There is no difference in the derived coefficients, but the uncertainty in the coefficients is much reduced when the data of Butler are added. The relative precision of the amplitudes in the Fourier decomposition (ratio of each amplitude to its uncertainty) is not so good as for photometry, but this is due to the incomplete phase coverage of the radial velocity data of Butler.

Surface brightness techniques require integration of the radial velocity curve to obtain differences in radii in linear units, which then are matched to angular diameters derived from photometry, yielding the distance. Improved radial velocity data should yield more precise distance estimates. For Eta Aquilae the addition of Butler's data changes the radial velocity curve very little, and the distance is unchanged. But for $\mathrm{X}$ Cygni the data of Butler greatly improve the radial velocity curve, and an absolute magnitude of -5.06 is obtained, 0.40 mag fainter than the result found otherwise. 